\title{
Development and validation of nanobodies specific to the oncogenic phosphatase Protein Tyrosine Phosphatase 4A3 (PTP4A3 or PRL-3)
}

Caroline N. Smith ${ }^{1}$, Min Wei ${ }^{1}$, Zachary A. Williamson ${ }^{1}$, Yelena Chernyavskaya ${ }^{1}, \mathrm{~K}$. Martin Chow ${ }^{1}$, Louis B. Hersh ${ }^{1}$, Konstantin V. Korotkov ${ }^{1}$, Jessica S. Blackburn ${ }^{1,2, *}$

${ }^{1}$ Department of Molecular and Cellular Biochemistry, University of Kentucky, Lexington, Kentucky, United States

2 The University of Kentucky Markey Cancer Center, Lexington, Kentucky, United States

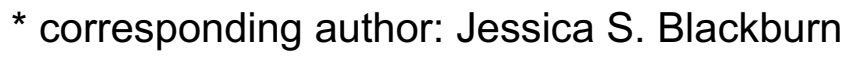

Email: jsblackburn@uky.edu

Running title: Nanobodies specific to PRL-3

Keywords: single-domain antibody (sdAb), phage display, antibody engineering, protein purification, confocal microscopy 


\begin{abstract}
Protein Tyrosine Phosphatase 4A3 (PTP4A3 or PRL-3) is an oncogenic dual specificity phosphatase that drives tumor metastasis, promotes cancer cell survival, has been linked to poor patient prognosis in a variety of tumor types. The mechanisms by which PRL-3 promotes tumor progression are not well understood, which is in part due to lack of tools to study this protein. The development of small molecule inhibitors that are specific to PRL-3 has proven difficult, as the PRL family is $>80 \%$ homologous. As research tools, antibodies directed against PRL-3 have been more successful, although they are limited to certain assay types. To address this, we have designed, purified, and tested alpaca-derived PRL-3 single domain antibodies, or nanobodies. We have identified 7 unique nanobodies that bind to PRL-3 in ELISA with no activity towards PRL-1 and PRL-2. Anti-PRL-3 nanobodies immunoprecipitated PRL-3 from cell lysates and were used to define PLR-3 localization in human colon cancer cells in immunofluorescence cell staining experiments. These anti-PRL-3 nanobodies represent an important expansion of the tool set used for the study of PRL-3, and can be used to better define the role of PRL-3 in cancer progression while serving as a useful first step in the development of biologics to target PRL-3 in the clinic.
\end{abstract}




\section{Introduction}

The Protein Tyrosine Phosphatase 4A (PTP4A) family of three proteins, also known as Phosphatases of Regenerating Liver (PRLs), are dual specificity phosphatases that act as oncogenes in multiple cancer types. PRL-3, in particular, has been identified as a potential cancer biomarker(1). PRL-3 expression was first identified as significantly upregulated in metastatic colorectal cancer, compared to primary lesions and normal tissue (2); since, PRL-3 has been implicated in progression and metastasis in gastric(3), ovarian(4), breast(5), brain(6), and prostate(7) cancers, melanoma(8)(9), and leukemias(10)(11). Experimental evidence indicates that PRL-3 expression increases proliferation, migration, and invasion of cancer cells in vitro(12)(13)(14) and enhances tumor growth and metastasis in mouse models(2)(15), while PRL-3 knockdown significantly suppresses tumor formation and spread in vivo(16). While overexpression of PRL-3 in tumors plays roles in inhibiting apoptosis, promoting epithelial to mesenchymal transition (EMT), and inducing migration, the mechanisms by which PRL-3 drives these processes and its physiological substrates remain unclear. The function of PRL-3 must be better defined if drugs targeting this protein are to be brought to the clinic.

The open questions regarding PRL-3, including its normal and cancerous function, localization, and substrate(s) are largely the result of insufficient tools to study this protein. The development of specific small molecule PRL-3 inhibitors has been difficult, as PRL proteins are highly homologous, and the PRL catalytic binding pocket is both shallow and hydrophobic(17). Currently, the most frequently used PRL inhibitors are the PRL-3 Inhibitor I (Sigma P0108), Analog 3(18), thienopyridone(19), and JMS-05334(20) all of which appear to inactivate PRLs via a redox reaction instead of directly binding with the protein's active site. Antibodies specific for PRL-3 have also proven difficult, with most antibodies lacking specificity towards PRL-3 over other PRL proteins. An antibody that has been validated by multiple research groups as specific for PRL-3 over PRL-1 and PRL-2(21)(19) was raised against the linear form of PRL-3 and cannot be used for studies assessing the native protein. A humanized monoclonal antibody, PRL-3-zumab, was recently developed and is shown to specifically bind to PRL-3 and have anticancer effects in vivo(22). The authors predicted that PRL-3 is capable of being presented on the cell surface through exosomal secretion, thereby allowing for the binding of PRL-3-zumab. This event stimulates Fc-receptor dependent interactions between PRL-3 positive cells and host immune effectors, activating classical antibody-mediated tumor clearance pathways leading to tumor cell death(22). However, while PRL-3-zumab is currently in phase 2 clinical trial (NCT04118114) for gastric and hepatocellular carcinomas, this antibody is not currently commercially available. Overall, research tools to study PRL-3 are lacking.

Single domain antibodies, or nanobodies, have recently emerged as immensely useful research tools and are likely to become useful therapeutics in a variety of diseases, including cancer $(23,24)$. Nanobodies were discovered in dromedaries, such as camels, llamas, and alpacas. These animals produce both antibodies with typical structure and those with an atypical structure that lacks light chains but have a similar variable region (VHH region) compared to conventional antibodies(25). The lack of light chains causes formation of a longer complementary determining region-(CDR)3 with a secondary disulfide bond(26) to stabilize nanobody structure. This shape permits formation of convex shapes, allowing nanobodies to reach narrow, concave binding and activation sites on proteins that normal antibodies cannot(27). Other advantages of nanobodies include their small size at $\sim 15 \mathrm{kDa}$, suggesting they may diffuse into membranes rather than requiring active transport, as well as their stability under stringent conditions, lack of immunogenicity, and a high specificity and affinity for their antigens(25).

There are many inherent properties of nanobodies that make them advantageous as therapeutics and specifically for cancer applications(28). The small size of nanobodies enables deep penetration into tumor tissue, and even the ability to cross the blood brain barrier(29), 
while maintaining low off-target effects(30). Nanobodies can also withstand high temperatures, elevated pressure, non-physiological pHs and denaturants, making them ideal research tools as well as useful therapeutic molecules(28). In 2019, the first nanobody therapeutic, Caplacizumab or Cablivi, was approved by the FDA to aid in accelerating platelet aggregation in acquired thrombotic thrombocytopenic purpura (aTTP), a disease that causes small blood clots throughout the body. There are currently 18 on-going clinical trials examining the efficacy of nanobodies across a range of cancer types(28).

Here, we describe the development and characterization of nanobodies against PRL-3. We show that the anti-PRL-3 nanobodies can be useful in biochemical assays such as ELISA, where they interact with PRL-3 with no binding to PRL-1 or PRL2. Anti-PLR-3 nanobodies can successfully and specifically immunoprecipitate PRL-3 protein from cell lysates and co-localize with PRL-3 in immunofluorescence assays in colorectal cancer cells. In all assays tested, nanobodies bound PRL-3 with high specificity over both PRL-1 and PRL-2. In total, the antiPRL-3 nanobodies are among the first tools in the PRL field that can specifically detect PRL-3 protein in a range of biochemical and cellular assays.

\section{Results}

\section{Alpaca derived anti-PRL-3 nanobodies exhibit varying amino acid sequences}

Human recombinant PRL-3 protein was injected into alpaca; B-lymphocytes expressing potential anti-PRL-3 nanobodies were harvested six weeks later and developed into a CDNA library, as diagramed in Figure 1A. Bacteriophage display panning of the library against recombinant PRL-3 and subsequent sequencing of the enriched clones identified 32 potential nanobodies, of which only 16 nanobodies contained a complete $\mathrm{N}$-terminal PelB sequence, Cterminal 6X-His-tag, and stop codon, and were without any undetermined amino acids (Figure 1B).

The anti-PRL-3 nanobody sequences were aligned. Sequences of nanobodies 91,90 , and 13 were identical throughout and were the most recurrent; therefore, we utilized nanobody 91 as our standard anti-PRL-3 nanobody throughout this study. Nanobodies were clustered based on their similarity to one another and their number of amino acid alterations or insertions based on nanobody 91 . These include four groups; 0, 1-2, 10-20, and 25+ amino acid changes when compared to nanobody 91 . We have proposed potential complimentary determining regions for these anti-PRL-3 nanobodies (Figure 1B) based on known structures of other nanobodies interacting with antigen(31).

\section{Anti-PRL-3 nanobodies are specific for PRL-3 over other PRL family members in protein assays}

PRL-1 and PRL-2 have $79 \%$ and $76 \%$ amino acid sequence homology to PRL-3(17), respectively, which has made the identification of specific small molecules and antibodies difficult. An indirect ELISA method was used to test the specificity of the anti-PRL-3 nanobodies towards PRL-3 over other PRL family members. Nanobodies and PRLs were purified from BL21 DE3 Star E. coli (Supplemental Figures 1 and 2), using nickel and size exclusion chromatography. The $\mathrm{N}$-terminal $6 \mathrm{X}$-His tag-was cleaved from purified PRL proteins, while the C-terminal $6 \mathrm{X}$-His-tag was left intact on nanobodies. PRL proteins were plated, and nanobody binding was detected through the addition of a secondary anti-His-HRP conjugated antibody.

All 16 nanobodies had a greater affinity for PRL-3 over PRL-1 and PRL-2 (Figure 2A), with most anti-PRL-3 nanobodies lacking any binding with PRL-1 or PRL-2 protein even in saturating conditions (Figure 2B-C). As expected, nanobodies with the same amino acid sequences had comparable binding to PRL-3. Five nanobodies produced less of a colorimetric shift following binding to PRL-3 either due to poor expression in E. coli (nanobodies 7, 68, and 
92) or low affinity for PRL-3 (nanobodies 23 and 28). Further studies focused on seven nanobodies $(4,10,16,19,26,84$, and 91$)$ with strong PRL-3 affinity and unique sequence both in the complimentary determining and framework regions.

\section{Anti-PRL-3 nanobodies do not inhibit the phosphatase activity of PRL-3}

$\mathrm{PRL}-3$ is a known oncogene, so specific targeting of PRL-3 to prevent function is very desirable. To determine if the nanobodies were capable of blocking PRL-3 phosphatase activity in in vitro assays, the ability of PRL-3/nanobody complexes to dephosphorylate a generic substrate, 6,8-Difluoro-4-Methylumbelliferyl Phosphate (diFMUP), was examined. Cleavage of a phosphate from diFMUP can induce a fluorescent signal at an excitation of $360 \mathrm{~nm}$ and emission at $460 \mathrm{~nm}$, which can be quantified. However, there was no significant difference in fluorescence between the PRL-3 in complex with nanobody compared to PRL-3 alone (Supplemental Figure 3 ), suggesting that the nanobody does not directly occlude the PRL-3 active site. However, with a molecular weight of 292, diFMUP is very small in comparison to potential PRL-3 protein substrates-whether the nanobodies might block PRL-3/substrate interaction at the active site to prevent PRL-3 mediated dephosphorylation events remains to be determined.

\section{Anti-PRL-3 nanobodies immunoprecipitated PRL-3 but not PRL-1 or PRL-2}

PRL-3 substrates remain largely undefined, in part due to insufficient tools for cell-based studies. The current commercially available PRL-3 antibodies have not been extensively validated for specificity towards PRL-3 over other PRLs. To address this, we tested the ability of the anti-PRL-3 nanobodies to identify PRL-3 protein in cell lysate. Anti-PRL-3 nanobodies were coupled to superparamagnetic Dynabeads $®$ M-270 Epoxy beads and used in immunoprecipitation of HEK293T cells expressing either 3XFLAG-PRL-1, -2, or -3. All nanobodies selectively pulled-down 3XFLAG-PRL-3 over PRL-1 and PRL-2, as assessed by anti-FLAG immunoblot (Figure 3A and Supplemental Figure 5). Some nanobodies were more specific to PRL-3 than others; nanobodies 4, 16, 19, and 84 pulled down small amounts of 3XFLAG-PRL-1 and/or PRL-2 (Figure 3A). Successful nanobody coupling to Dynabeads was confirmed by the presence of 6X-His-tag in all samples (Figure 3B). Beads alone do not immunoprecipitate 3XFLAG-PRL-3 (Supplemental Figure 4), indicating that the beads do not play a role in terms of immunoprecipitation. In total, anti-PRL-3 nanobodies 10, 26, and 91 can be used to specifically immunoprecipitate PRL-3, with no binding to PRL-1 or PRL-2.

\section{Anti-PRL-3 nanobodies specifically detect PRL-3 in fixed cells in immunofluorescence assays}

Our next goal was to determine if nanobodies could specifically identify PRL-3 in fixed cells, which can be useful for studies involving PRL-3 localization and trafficking. The human colon cancer cell line HCT116 was transfected with CMV:GFP-PRL constructs to visualize the PRLs and determine the extent to which the anti-PRL-3 nanobodies co-localize with each of them. Nanobody 91 completely co-localized with GFP-PRL-3, which was seen at both at the plasma membrane and in the nucleus (Figure 4), as previously described $(32,33)$. This nanobody did not bind with GFP-PRL-1, GFP-PRL-2, or the GFP control. All anti-PRL-3 nanobodies tested were similarly specific for PRL-3 over PRL-1 and PRL-2, although some nanobodies exhibited a higher non-specific background signal (Supplemental Figure 6).

\section{Discussion}

The PRL family of proteins have emerged as important players in cancer progression, with PRL-3 now recognized as an bona fide oncogene. However, the mechanisms by which PRL-3 promotes tumor growth and spread are largely unknown and are important to define before PRL-3 inhibitors can be widely used in patients. A major roadblock in understanding the 
role of PRL-3 in a cancer is a lack of tools with which to study this protein. While PRL antibodies and inhibitors have been used for research purposes, they often come with caveats. For example, the allosteric PRL-3 inhibitor JMS-053 equally targets all PRL-1, -2 , and $-3(20)$. The commercially available antibody that has been validated as specific towards PRL-3 was generated against denatured protein and cannot be used in assays that analyze native protein. PRL-3-zumab, which has been extensively validated as specific towards PRL-3, requires an in vivo microenvironment for its anti-cancer activity and has not been made widely available $(22,34)$.

Like PRL-3-zumab, the anti-PRL-3 nanobodies that we have generated are very specific to PRL-3 over PRL-1 and PRL-2 in vitro. However, nanobodies carry advantages over their conventional antibody counterparts in general and in terms of PRL-3. So far, PRL-3-zumab has been applied as an extracellular reagent, as it is hypothesized to bind PRL-3 on the cell surface to induce an immune response to kill cancer cells(22). While nanobodies are 10-fold smaller compared to conventional antibodies and are stable proteins, they are unlikely to penetrate cell membranes via passive diffusion. Recent efforts have been focused on developing cellpenetrating nanobodies. For example, Herce et al, attached intracellularly stable cyclic argininerich cell-penetrating peptides (CPPs) to nanobodies(35). CPPs have been previously shown to increase the size of cargo that can be delivered efficiently into living cells(36), and this strategy was successful in delivering nanobodies targeted against GFP (27.5 kDa), GFP-PCNA (63 $\mathrm{kDa}$ ) and the therapeutically relevant Mecp2- GFP fusion protein (83 kDa) into Hela cells to study protein interactions(35). Similar technologies could be used to deliver anti-PRL-3 nanobodies for studies on PRL-3 localization or function in living cells. Nanobodies have also emerged as a useful tool to neutralize target proteins involved in disease (37-40)—delivery of anti-PRL-3 nanobodies into the cell would position it as a useful biological therapeutic in a variety of cancers.

A major goal in the PRL field is the completion of in-depth structural studies of PRL-3, especially in complex with current inhibitors. This information can give insight into how to design better small molecules to target PRL-3, as well as identification of useful sites to target on the protein. However, PRL-3 has been difficult to crystalize as it can move between open and closed conformation (17). Current PRL-3 antibodies are not practical to stabilize PRL-3 for crystallization, as they are large, glycosylated, multi-domain proteins, which are not suitable for applications such as X-ray crystallography. The anti-PRL-3 nanobodies were successful in both PRL-3 ELISA and immunoprecipitation of PRL-3, suggesting that they bind to the protein very well. The small size, high stability, and high specificity of nanobodies lends them well towards acting as chaperones in structural studies(31), and our data suggest that the anti-PRL-3 nanobodies may be useful to stabilize the PRLs in a single conformation to aid in crystallization studies.

In summary, we have developed alpaca-derived single domain antibodies against PRL3 , and showed that they can specificity detect PRL-3 in purified protein assays, in human cell lysates, and in situ in fixed cancer cells, while they do not interfere with PRL-3 phosphatase activity. Nevertheless, these nanobodies begin to fill an important gap in the tools needed to study PRL-3 function in normal physiology and cancer and have the potential to provide valuable insight into PRL-3 substrates, trafficking, structure, and inhibition. 


\section{Materials and Methods}

\section{Plasmids and other reagents}

To generate protein for alpaca immunization, human PRL-3 cDNA was amplified with gene specific primers and cloned into the bacterial expression vector $\mathrm{pET} 28 \mathrm{~b}$ provided by Dr. Konstatin Korotkov (University of Kentucky, Lexington, KY 40536) at Nhel and Xhol restriction sites using T4 ligase. Recombinant PRL-1, -2 , and -3 were made in the same fashion for all subsequent assays

The 3XFLAG-tagged PRL mammalian expression plasmids were made by cloning PCR products of full length PRL-1, -2 , or -3 human CDNA into p3XFLAG-CMV-14 expression vector (Sigma, E7908). Then 3X-FLAG-PRLs were cloned into plenti-CMV-puro (Addgene 17452) to make plenti-CMV-3XFLAG-PRL-puro constructs.

The GFP-tagged PRL overexpressing plasmids were made by cloning full length PRL-1, -2 , or 3 gBlocks $^{\text {TM }}$ Gene Fragments (IDT) into the pcDNA ${ }^{\mathrm{TM}} 3.1$ (-) (Invitrogen V79520) at BamHI and Hindlll restriction sites. A GFP gBlock was subsequently cloned into each of the pCDNA3.1PRL plasmids to generate CMV:GFP-PRL fusion constructs at Notl and BamHI restriction sites.

\section{Production, panning, and sequencing of nanobodies}

Nanobodies were produced by the University of Kentucky Protein Core, as previously described(31). Briefly, 100 $\mu \mathrm{g}$ of recombinant PRL-3 antigen was subcutaneously injected into alpacas once per week for six weeks to boost nanobody presence in the immune system. 3-5 days following the final injection $50 \mathrm{~mL}$ of alpaca blood was harvested to isolate peripheral blood lymphocytes by density gradient centrifugation. RNA was isolated and cDNA was synthesized using reverse transcriptase, to generate bacteriophage display cDNA library by cloning with restriction enzymes into the phage display vector pMES4 followed by the expression of the insert fused to gene III of the filamentous phage for the production of the phage solution. Two rounds of phage display utilizing this cDNA library yielded 32 potentially $\mathrm{VHH}$ positive clones that were analyzed for sequencing using the primer $p E X-R e v$ (CAGGCTTTACACTTTATGCTTCCGGC). DNA sequences were translated using the ExPASy Bioinformatics Resource Portal Translate Tool (https://web.expasy.org/translate/) where they were analyzed for nanobody components including pelB sequence and 6X-His-tag followed by a stop codon. 16 of 32 clones embodied all of these components and were carried through to following experiments.

\section{Cell lines and cell culture}

All human cell lines used in this study (HEK293T, HCT116) were authenticated by short tandem repeat (STR) profiling and tested for mycoplasma contamination prior to experiments. HEK293T (ATCC CRL-3216) and HCT116 (ATCC CCL-247) cells were grown in 1X DMEM (Thermofisher, 11965092). For all, media were supplemented with $10 \%$ heat-inactivated fetal bovine serum (R\&D Systems, $\mathrm{S} 11150 \mathrm{H}$, Lot. $\mathrm{H} 19109$ ). Cells were cultured at $37^{\circ} \mathrm{C}$ with $5 \% \mathrm{CO}_{2}$. To overexpress the CMV:GFP-PRL and CMV:3XFLAG-PRL plasmids, cells were transfected using Lipofectamine 3000 (Thermofisher, L3000-015) following the manufacturer's protocol.

\section{Protein purification}

pET28b-PRL and pMES4-Nanobody expression plasmids described above were transformed into and expressed using the One Shot BL21 Star DE3 bacterial cell line (Invitrogen, C601003) by stimulating induction with $0.5 \mathrm{mM} \mathrm{IPTG}$ (Fisher Scientific, BP175510) for 16 hours at $16^{\circ} \mathrm{C}$ 
following a culture O.D.600 of 0.6. Cells were pelleted at 5,000 rpm for 15 minutes at $4^{\circ} \mathrm{C}$ and resuspended in $10 \mathrm{~mL}$ of lysis buffer [300 mM NaCl (VWR BDH9286), 20mM Tris pH 7.5, 10 mM Imidazole pH 8.0 (Sigma-Aldrich I2399), 1:1000 protease inhibitor cocktail (Sigma-Aldrich P8465)] per gram of cell pellet and lysed using a microfluidizer (Avestin, EmulsiFlex-C5). Debris was pelleted at $18,000 \mathrm{rpm}$ for 50 minutes at $4^{\circ} \mathrm{C}$ and lysate was run over $1 \mathrm{~mL}$ columns (Biorad, 7321010) packed with Ni-NTA Resin (VWR, 786-940). PRLs were eluted with $2 \mathrm{~mL}$ of elution buffer ( $300 \mathrm{mM} \mathrm{NaCl}, 20 \mathrm{mM}$ Tris pH 7.5, and $250 \mathrm{mM}$ Imidazole pH 8.0). Nanobodies underwent two elution steps, the first with $30 \mathrm{mM}$ Imidazole elution buffer, and the second with $250 \mathrm{mM}$ Imidazole elution buffer. The N-terminal 6X-His-tag on recombinant PRLs was cleaved using TEV protease (gift from Konstantin Korotkov), and samples were reapplied to Ni-NTA column to remove uncleaved protein as well as TEV. Recombinant nanobodies remained with their C-terminal 6X-His-tag intact. All samples underwent buffer exchange to remove imidazole (300 mM NaCl, $20 \mathrm{mM}$ Tris pH 7.5) and were further purified using a Superdex 200 Increase 10/300 GL column (GE, 28990944) on an ÄKTA purification system in buffer containing $100 \mathrm{mM}$ $\mathrm{NaCl}$ and $20 \mathrm{mM}$ HEPES (Fisher Scientific, BP310-100) pH 7.5. Purification was verified by running samples on 4-20\% Mini-PROTEAN TGX Stain-Free Gels (Biorad 4568094). The purest fractions were pooled, concentrated together, flash frozen on dry ice, and stored at $-80^{\circ} \mathrm{C}$.

\section{ELISA for Nanobody/PRL binding specificity}

Recombinant, purified, PRL-1, -2 , and -3 were plated at $1 \mu \mathrm{g} / \mathrm{mL}(100 \mu \mathrm{l})$ in Sodium Bicarbonate Buffer [0.42g Sodium Bicarbonate (Fisher Scientific, BP328-500) in 50mL diH $\mathrm{d}_{2} \mathrm{O}$ in Corning ${ }^{\circledR}$ 96 Well EIA/RIA Assay Microplates (Sigma, CLS3590) and incubated for $16-20$ hours at $4^{\circ} \mathrm{C}$. Plates were washed three times with $0.05 \%$ PBST and loaded with a blocking solution of $0.5 \%$ BSA (Fisher Scientific, BP9706100) in 0.1\% PBST for 1 hour at room temperature. Blocking buffer was removed and nanobodies were diluted to $1 \mu \mathrm{g} / \mathrm{mL}$, or designated concentration for dosing experiments, and incubated in wells for 1 hour at room temperature. Wells were washed 3 times in PBS and incubated with 1:1000 anti-His HRP antibody (GenScript, A00612, Lot. 19K001984), for 1 hour at room temperature. Plates were washed 3 times with PBS and developed with TMB 2-Component Microwell Peroxidase Substrate Kit (Seracare, 5120-0053). Reactions were stopped after 90 seconds with $0.1 \mathrm{~N} \mathrm{HCl}$ (Fisher Scientific, A144500) and read on a Biotek Synergy Multi-mode Plate Reader at $450 \mathrm{~nm}$. Controls included PRL only wells, to specify lack of a 6X-His-tag, nanobody and secondary only wells to specify the necessity of PRL presence for binding, and buffer only to provide evidence that sodium bicarbonate and BSA could not elucidate a colorimetric change. Raw data from all control wells was pooled for each plate, and experimental wells were normalized to controls by dividing individual wells by average control wells. Individual well readouts were then placed in Prism 7 in Grouped format Table, where values for two replicate experiments were graphed for Relative Absorbance at $450 \mathrm{~nm}$ compared to average of control wells.

\section{Nanobody Coupling to Dynabeads}

PRL-3 nanobodies were coupled to Dynabeads (Life Technologies, 14311D) for downstream 3XFLAG-PRL-3 immunoprecipitation following manufacturer's instructions. Briefly, $150 \mu \mathrm{g}$ of nanobody protein supplemented with $\mathrm{C} 1$ buffer to $250 \mu \mathrm{L}$ was added to $5 \mathrm{mg}$ of Dynabeads $\circledR$ M-270 Epoxy beads after the beads were washed with $1 \mathrm{~mL}$ of $\mathrm{C} 1$ buffer. Then, $250 \mu \mathrm{L}$ of C2 buffer was added to the beads and nanobody mixture to incubate on a rotator at room temperature overnight (16-24 hours). After removing the supernatant, the nanobody-coupled beads were washed subsequently with HB (0.05\% Tween 20$)$, and LB $(0.05 \%$ Tween 20$)$ buffer once, SB buffer shortly twice and SB buffer for 15 minutes once. Finally, the nanobody-coupled beads were resuspended in $500 \mu \mathrm{L} \mathrm{SB}$ buffer and stored at $4^{\circ} \mathrm{C}$ prior to experimentation. 


\section{Immunoprecipitation of PRL-3 with nanobody coupled to Dynabeads}

HEK293T cells ( 20 million) were lysed for 30 minutes with intermittent vortexing in Pierce IP lysis buffer (Thermo 87788 ) supplemented with $1 \%$ protease inhibitor cocktail (IP buffer) at 500 $\mu \mathrm{l}$ per 10 million cells and spun at $12,000 \mathrm{rpm}$ for 10 minutes at $4^{\circ} \mathrm{C}$ to pellet cell debris. Protein concentration was quantified using the Quick Start Bradford 1X Dye Reagent (Biorad, 5000205). $150 \mu \mathrm{L}$ of nanobody-coupled beads were washed in $1 \mathrm{~mL}$ of PBS for 5 minutes, precipitated, then equilibrated in $500 \mu \mathrm{L}$ of IP buffer for 5 minutes. $2.5 \mathrm{mg}$ of total extracted protein was added to the balanced nanobody-beads complex for incubation with rocking at $4{ }^{\circ} \mathrm{C}$ overnight. After washing the beads-protein complex in cold PBS four times, $50 \mu \mathrm{L} \mathrm{2x}$ Laemmli Sample Buffer (Biorad, 161-0737) with 2-Mercaptoethanol (Fisher Scientific, 03446I-100) was added to the beads, the mixture was boiled at $95^{\circ} \mathrm{C}$ for 10 minutes, and the supernatant was collected for western blot analysis.

\section{Western Blot}

$30 \mu \mathrm{g}$ of total protein for input or the pulldown supernatant was loaded into a 4-20\% MiniPROTEAN® TGX Stain-Free ${ }^{\text {TM }}$ Protein Gels. Total protein was assessed through stain free imaging on Biorad ChemiTouch Imaging System, which allows use of total protein as the loading control. Protein was transferred onto PVDF membrane (Biorad, 162-0255) using the Trans-Blot Turbo Transfer System (Biorad 1704150). Membranes were blocked with 5\% milk in $0.1 \%$ TBST for 1 hour and probed with one of the following antibodies at the designated dilution overnight at $4^{\circ} \mathrm{C} .1: 3000$ Monoclonal ANTI-FLAG $₫$ M2 antibody (Sigma, F1804, Lot.

SLBK1346V) or 1:1000 anti-His HRP antibody (GenScript, A00612, Lot. 19K001984). Following three washes with $0.1 \%$ TBST, secondary HRP-conjugated anti-mouse IgG antibody (Cell Signaling, 7076S, Lot. 33) was added at 1:2500 for 1 hour and membranes were imaged using Clarity Western ECL Substrate (Biorad, 1705061).

\section{Immunofluorescence in Fixed GFP-PRL cells with nanobodies}

Transfected cells were plated at 5,000 cells per well in 96-well black glass bottomed plates (Cellvis, P96-1.5H-N). All solution exchanges and imaging occurred in the 96-well plate. 24 hours post-transfection cells were fixed in 4\% paraformaldehyde (VWR, AAJ61899-AK) for 15 minutes, rinsed in PBS, permeabilized for 10 minutes in 1\% 100X Triton (Sigma, X100-100), and rinsed in PBS. Blocking solution of 2\% BSA in PBS was applied for 1 hour to all wells. All nanobodies were diluted to $1 \mathrm{mg} / \mathrm{ml}$ in blocking solution, and further diluted 1:1000 and incubated with the cells for 1 hour at room temperature followed by five PBS washes. Detection was carried out using an anti-alpaca IgG VHH conjugated to Alexa Fluor-594 (Jackson ImmunoResearch, 128-585-232) diluted 1:400 in blocking solution, and counterstained with Hoechst, 1:1000 dilution (ThermoFisher, H3570). All wells were washed in PBS five times prior to imaging. Images were acquired with a Nikon A1R confocal using the 40X water objective. Images were processed in Adobe Photoshop 2020 to both increase image brightness, overlay the 405 (Hoescht), 488 (GFP-PRL-3), and 561 (Nanobodies) channels. Channels were pseudocolored by RGB channels.

\section{Phosphatase Assay}

$2.5 \mu \mathrm{M}$ of recombinant PRL-1, -2 , or -3 was mixed with $2.5 \mu \mathrm{M}$ of each nanobody in black 384well plates (Thermo Scientific, 164564), and incubated at room temperature for 1 hour in Reaction Buffer $(20 \mathrm{mM}$ Tris, $150 \mathrm{mM} \mathrm{NaCl})$. Following incubation, the recombinant protein mixtures were combined with $12.5 \mu \mathrm{M}$ diFMUP (Life Technologies, E12020), added to 384-well plates, and incubated for 20 minutes in the dark at room temperature. Fluorescence intensities were measured on a Biotek Synergy Multi-mode Plate Reader at $360 \mathrm{~nm} / 460 \mathrm{~nm}$ excitation and emission receptively. Raw values for non-substrate containing controls were averaged and 
substracted from values of wells incubated with substrate to remove background fluorescence. Raw values were transferred to Prism 7 in Grouped format where two replicate experiments were combined for final data processing.

\section{Figures}

A

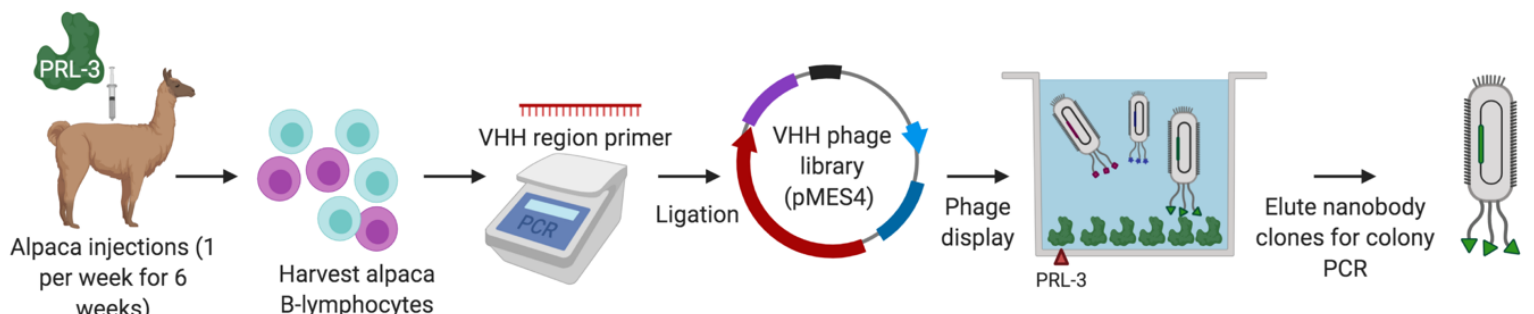

B PelB sequence

Harvest alpaca

PRL-3

CDR2

CDR3

NB13 MKYLLPTAAAGLLLLAAQPAMAQVQLQESGGGLVQTGGSLRLSCAASQSTFNFDVMGWYRLAPGKQREFLTSITNGGNIYYADSVKGRFTIARDDSKTTMYLEMNSLKPEDTAVYTCYGQTHKPRVTTSWGQGTQVTVSSHHHHHHNB90 MKYLLPTAAAGLLLLAAQPAMAQVQLQESGGGLVQTGGSLRLSCAASQSTFNFDVMGWYRLAPGKQREFLTIITNGGNIYYADSVKGRFTIARDDSKTTMYLEMNSLKPEDTAVYTCYGQTHKPRVTTSWGQGTQVTVSSHHHHHH-

NB4 MKYLLPTAAAGLLLLAAQPAMAQVQLQESGGGLVQTGGSLRLSCAASQSTYNFDVMGWYRLAPGKQREFLTSITNGGNIYYADSVKGRFTIARDDSKTTMYLEMNSLKPEDTAVYTCYGQTHKPRVTTSWGQGTQVTVSSHHHHHHNB7 MKYLLPTAAAGLLLLAAQPAMAQVQLQESGGGLVQTGGSLRLSCAASQSTFNFYVLGWYRLAPGKQREFLTSITNGGNIYYADSVKGRFTIARDDSKTTMYLEMNSLKPEDTAVYTCYGQTHKPRVTTSWGQGTQVTVSSHHHHHHNB10 MKYLLPTAAAGLLLLAAQPAMAQVQLQESGGGLVQTGGSLRLSCAASQSTFNFNVMGWYRLAPGKQREFLPSLTNGGNIYYADSVKGRFTIARDDSKTTTYLEMNSLKPEDTAVYTCYGQTHKPRVTTSWGQGTQVTVSSHHHHHHNB16 MKYLLPTAAAGLLLLAAQPAMAQVQLQESGGGLVQTGGSLRLSCAASQSTFNFDVMGWYRLAPGKQREFLTSITNGGNIYYADSVKGRFTTARDDSKTTMYLEMNSLKPEDTAVYTCYGQTHKPRVTTSWGQGTQVTVSSHHHHHHNB18 MKYLLPTAAAGLLLLAAQPAMAQVQLQESGGGLVQTGGSLRLSCAASQSTFNFDVMGWYRLAPGKQREFLTIITNGGNIYYADSVKGRFTTARDDSKTTMYLEMNSLKPEDTAVYTCYGQTHKPRVTTSWGQGTQVTVSSHHHHHHNB29 MKYLLPTAAAGLLLLAAQPAMAQVQLQESGGGLVQTGGSLRLSCAASQSTYNFDVMGWYRLAPGKQREFLTSITNGGNIYYADSVKGRFTIARDDSKTTMYLEMNSLKPEDTAVYTCYGQTHKPRVTTSWGQGTQVTVSSHHHHHH-

NB19 MKYLLPTAAAGLLLLAAQPAMAQVQLQESGGGLVQAGGSLGLSCAASRSIFNFKVMGWYRQAPGKQRELVASITNSDNTYYADSVKGRFTISREDAKTTMYLEMNSLKPEDTAVYRCYGQNWGLRATTYWGQGTQVTVSSHHHHHHNB84 MKYLLPTAAAGLLLLAAQPAMAQVQLQESGGGLVQAGGSLGLSCAASRSIFNFKVMGWYRQAPGKQRELVASITNSDNTYYADSVKGRFTISREDAKTTMYLEMNSLKPEDTTVYRCYGQNWGLRATTYWGQGTQVTVSSHHHHHHNB92 MKYLLPTAAAGLLLLAAQPAMAQVQLQESGGGLVHTGGSLILSCAASRSFFIFDVMGWYRQAPGNHREFVTSITNGGNVYYVDSVKGRFNIPKDDSNTTMYLEMNSLKPEDTAVYTCYGQIHKPRVTTSWGQGTQVTVSSHHHHHH-

NB23 MKYLLPTAAAGLLLLAAQPAMAQVQLQESGGGLVQAGGSLRLSCLGSGISVSVNGVAWYRLAPGKQRERVALITTDNATTYADSVKGRFAISRDKIKNXVYLQMSDLKPEDXAVYYCNEVNXLGYFTNYWGQGTQVTVSSHHHHHH-

NB26 MKYLLPTAAAGLLLLAAQPAMAQVQLQESGGGLVQPGGSLKLSCVVTGLHYYTEGWFRQAPGKEREGVSCISSSDGRTDYIDSVKGRFTISEDNDNKTVYLQMNTLKPDDTGVYYCVAERGPRGSTWWETYDYWGQGTHVTVSSHHHHHH-

NB28 MKYLLPTAAAGLLLLAAQPAMAQVQLQESGGGSVQPGGSLRLSCSASGRTSSMYAMGWFRQAPGKEREFVAGIRWSVGTTSYADSVKGRFTISRDNAENTVYLQMNSLKPEDTAVYYCAAGTPIVLSSSRYAYWGQGTQVTVSSHHHHHH-

NB68 MKYLLPTAAAGLLLLAAQPAMAQVQLQESGGGLVQAGGSLRLSCSASGRTSSMYAMGWFRQAPGKEREFVAGIRWSVGTTSYADSVKGRFTISRDNAENTVYLDMNALKSEDTAMYYCTNDSGRPRSQGTQVTVSSHHHHHH-

Figure 1: Isolation of PRL-3 specific nanobodies. A - Schematic demonstrating the process completed from initial recombinant PRL-3 injection in alpacas to colony PCR to reach sequence identification of potential anti-PRL-3 nanobodies (Created with BioRender.com). B - Amino acid sequence for 16 nanobodies that showed positive result in colony PCR and in sequencing with Eurofins primer pexR. NB91 was the most common sequence in our pool of clones, demonstrating necessary nanobody components with PelB (directs protein to the bacterial periplasm during expression), and expected complimentary determining regions. Each group of nanobodies is either the same sequence as NB91, differs by 1-2, 10-20, or 25+ amino acids. 


\section{A Relative Absorbance of His-tag antibody following TMB-peroxidase addition}
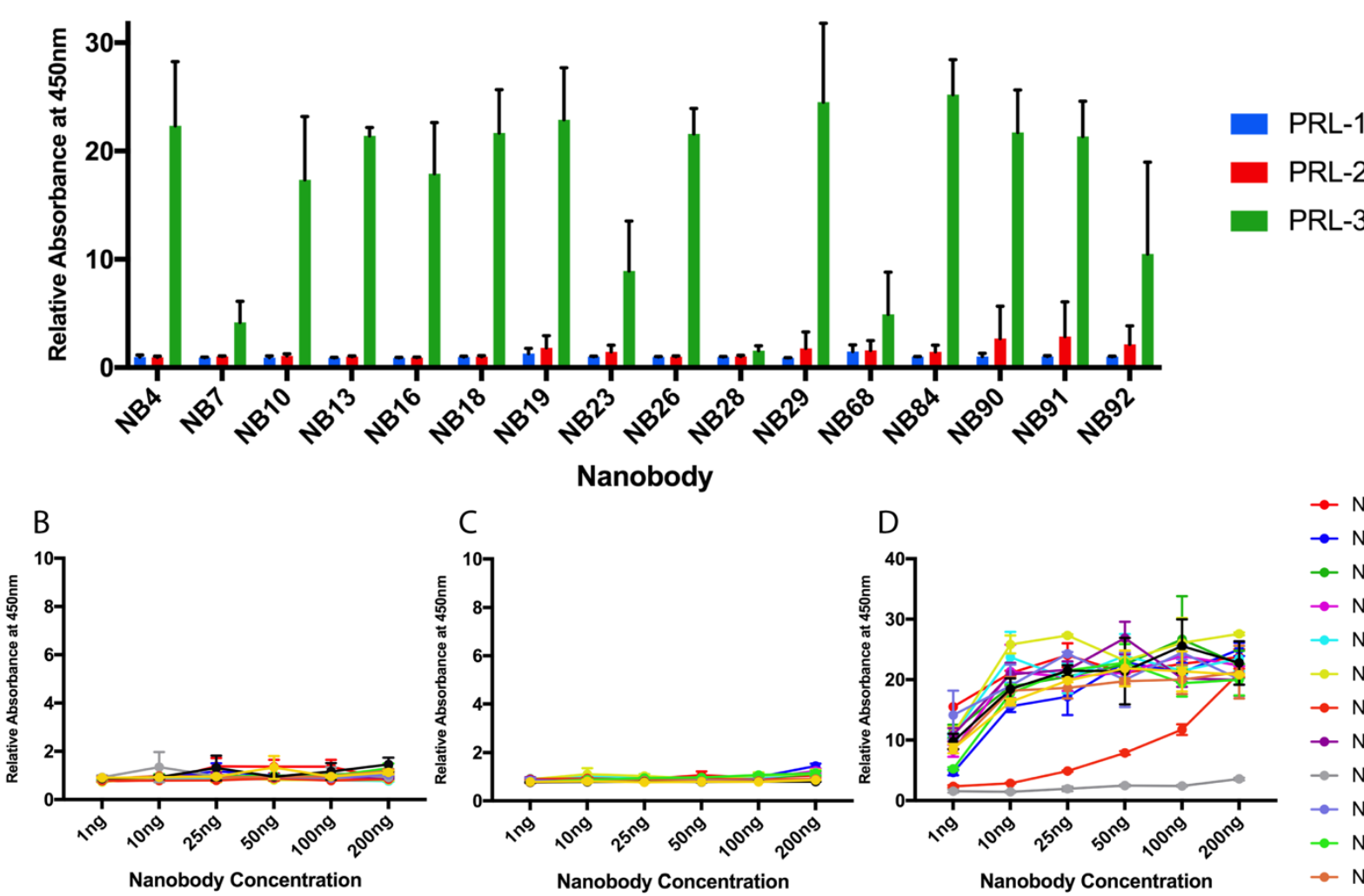

$\rightarrow$ NB4

$\rightarrow$ NB10

$\rightarrow$ NB13

$\rightarrow$ NB16

$\rightarrow$ NB18

$\rightarrow$ NB19

$\rightarrow$ NB23

$\rightarrow$ NB26

$\rightarrow$ NB28

$\rightarrow$ NB29

$\rightarrow$ NB84

$\rightarrow$ NB86

$\rightarrow$ NB90

$\rightarrow$ NB91

Figure 2: Nanobodies are specific for PRL-3 over the other PRL family members. A ELISA utilizing 96-well plates coated with 100ng of the PRLs, probed with 100ng of each nanobody followed by 1:1000 anti-His HRP antibody (Genscript). ELISA of 100ng of each B PRL-1, C - PRL-2, D - PRL-3 saturated with up to 200 ng of 16 nanobodies. ELISAs were developed for 1.5 minutes with $100 \mu$ TMB 2-Component Microwell Peroxidase Substrate Kit (Seracare) and stopped with $100 \mu \mathrm{l} 0.1 \mathrm{~N} \mathrm{HCl}$, and read at $450 \mathrm{~nm}$. All assays were completed with two technical replicates and repeated in two biological replicates. 

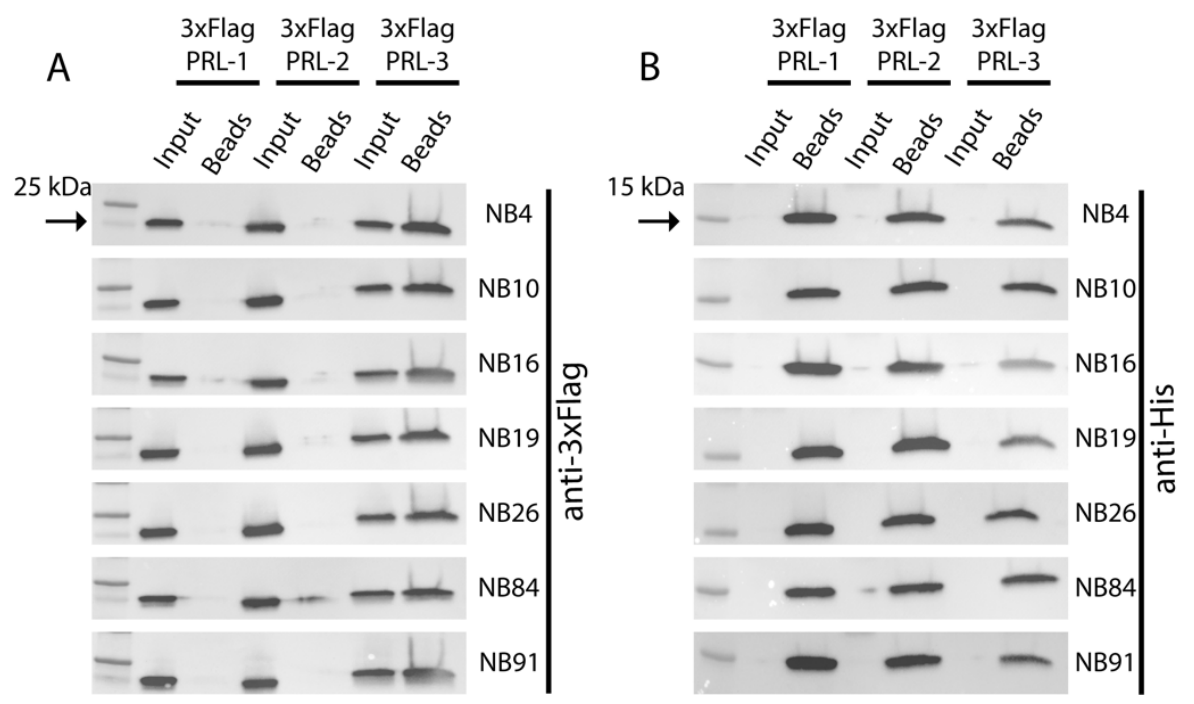

Figure 3: Nanobodies can be used to immunoprecipitate overexpressed PRL-3. PRL-3 specific nanobodies coupled to superparamagnetic Dynabeads ${ }^{\circledR}$ M-270 Epoxy beads were used in immunoprecipitation assays with lysates from HEK293T cells transduced with 3xFLAGPRL-1, -2 or -3. A - All nanobodies pull down 3xFLAG-PRL-3 with little to no pull down of 3xFLAG-PRL-1 or 3XFLAG-PRL-2. B - Successful nanobody coupling to Dynabeads in all groups was verified using an antibody against the $\mathrm{C}$-terminal $6 \mathrm{XHis}$-tag present on each nanobody. 

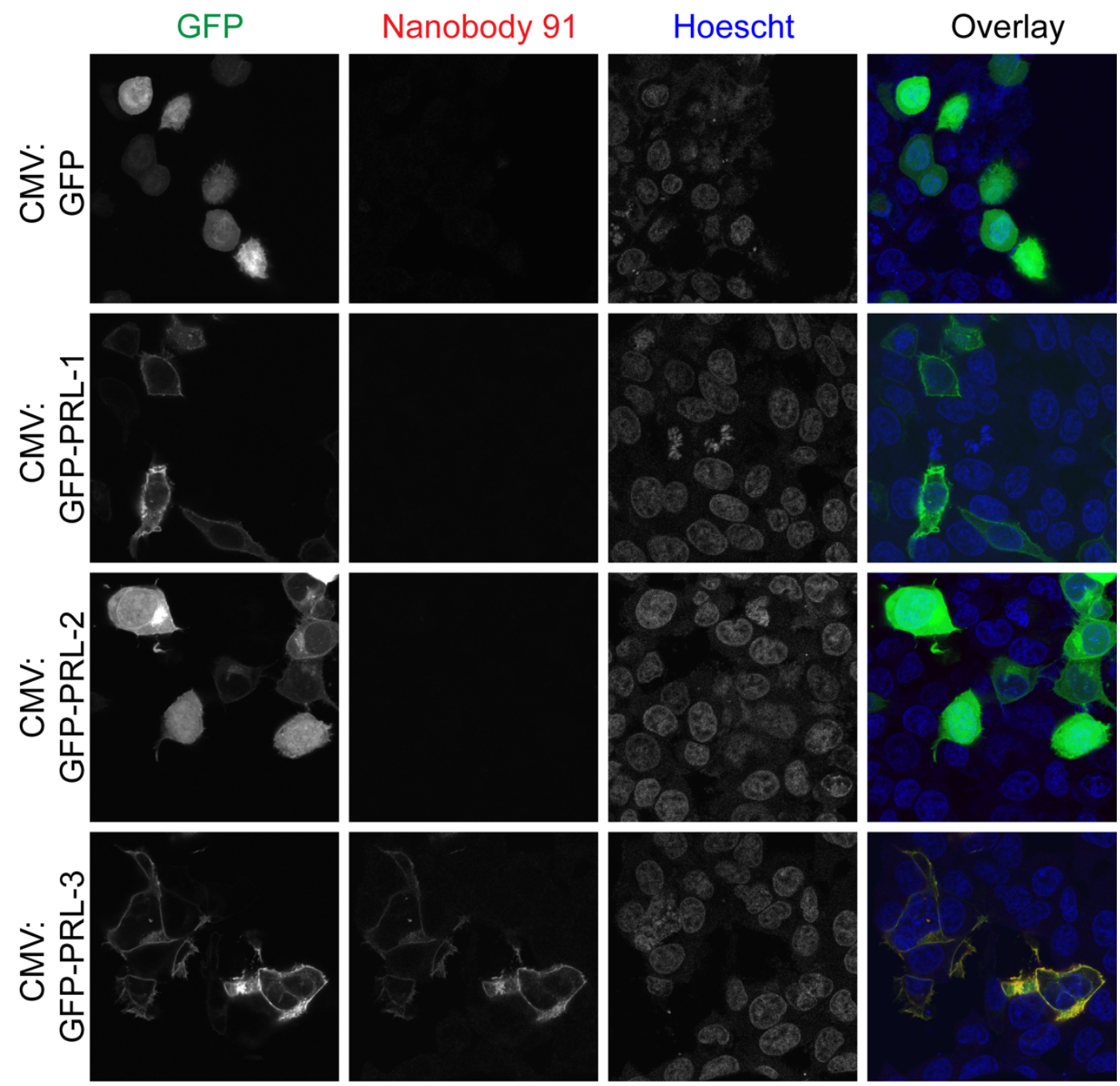

Figure 4: Nanobodies are specific to PRL-3 in immunofluorescence assays. HCT116 colorectal cancer cells were transfected with CMV:GFP, CMV:GFP-PRL-1, CMV:GFP-PRL-2, or CMV:GFP-PRL-3 for 24 hours prior to cell fixation and permeabilization. Immunofluorescence assays were completed with $1: 10001 \mathrm{mg} / \mathrm{mL}$ NB91 followed by 1:400 Alexa Fluor® 594AffiniPure Goat Anti-Alpaca lgG, VHH domain and show nanobodies detect PRL-3 but not PRL1 or PRL-2. 


\section{References}

1. Tonks, N. K. (2013) Protein tyrosine phosphatases--from housekeeping enzymes to master regulators of signal transduction. FEBS J 280, 346-378

2. Saha, S., Bardelli, A., Buckhaults, P., Velculescu, V. E., Rago, C., St Croix, B., Romans, K. E., Choti, M. A., Lengauer, C., Kinzler, K. W., and Vogelstein, B. (2001) A phosphatase associated with metastasis of colorectal cancer. Science 294, 1343-1346

3. Dai, N., Lu, A. P., Shou, C. C., and Li, J. Y. (2009) Expression of phosphatase regenerating liver 3 is an independent prognostic indicator for gastric cancer. World J Gastroenterol 15, 1499-1505

4. Ren, T., Jiang, B., Xing, X., Dong, B., Peng, L., Meng, L., Xu, H., and Shou, C. (2009) Prognostic significance of phosphatase of regenerating liver-3 expression in ovarian cancer. Pathol Oncol Res 15, 555-560

5. den Hollander, P., Rawls, K., Tsimelzon, A., Shepherd, J., Mazumdar, A., Hill, J., Fuqua, S. A., Chang, J. C., Osborne, C. K., Hilsenbeck, S. G., Mills, G. B., and Brown, P. H. (2016) Phosphatase PTP4A3 Promotes Triple-Negative Breast Cancer Growth and Predicts Poor Patient Survival. Cancer Res 76, 1942-1953

6. Wang, L., Liu, J., Zhong, Z., Gong, X., Liu, W., Shi, L., and Li, X. (2016) PTP4A3 is a target for inhibition of cell proliferatin, migration and invasion through Akt/mTOR signaling pathway in glioblastoma under the regulation of miR-137. Brain Res 1646, 441-450

7. Vandsemb, E. N., Bertilsson, H., Abdollahi, P., Storkersen, O., Vatsveen, T. K., Rye, M. B., Ro, T. B., Borset, M., and Slordahl, T. S. (2016) Phosphatase of regenerating liver 3 (PRL3 ) is overexpressed in human prostate cancer tissue and promotes growth and migration. J Transl Med 14, 71

8. Fang, X. Y., Song, R., Chen, W., Yang, Y. Y., Gu, Y. H., Shu, Y. Q., Wu, X. D., Wu, X. F., Sun, Y., Shen, Y., and Xu, Q. (2015) PRL-3 Promotes the Malignant Progression of Melanoma via Triggering Dephosphorylation and Cytoplasmic Localization of NHERF1. J Invest Dermatol 135, 2273-2282

9. Johansson, J. A., Marie, K. L., Lu, Y., Brombin, A., Santoriello, C., Zeng, Z., Zich, J., Gautier, P., von Kriegsheim, A., Brunsdon, H., Wheeler, A. P., Dreger, M., Houston, D. R., Dooley, C. M., Sims, A. H., Busch-Nentwich, E. M., Zon, L. I., Illingworth, R. S., and Patton, E. E. (2020) PRL3-DDX21 Transcriptional Control of Endolysosomal Genes Restricts Melanocyte Stem Cell Differentiation. Dev Cell 54, 317-332 e319

10. Wei, M., Haney, M. G., Rivas, D. R., and Blackburn, J. S. (2020) Protein tyrosine phosphatase 4A3 (PTP4A3/PRL-3) drives migration and progression of T-cell acute lymphoblastic leukemia in vitro and in vivo. Oncogenesis 9, 6

11. Zhou, J., Chong, P. S., Lu, X., Cheong, L. L., Bi, C., Liu, S. C., Zhou, Y., Tan, T. Z., Yang, H., Chung, T. H., Zeng, Q., and Chng, W. J. (2014) Phosphatase of regenerating liver-3 is regulated by signal transducer and activator of transcription 3 in acute myeloid leukemia. Exp Hematol 42, 1041-1052 e1041-1042

12. Al-Aidaroos, A. Q., Yuen, H. F., Guo, K., Zhang, S. D., Chung, T. H., Chng, W. J., and Zeng, Q. (2013) Metastasis-associated PRL-3 induces EGFR activation and addiction in cancer cells. J Clin Invest 123, 3459-3471 
13. Peng, L., Xing, X., Li, W., Qu, L., Meng, L., Lian, S., Jiang, B., Wu, J., and Shou, C. (2009) PRL-3 promotes the motility, invasion, and metastasis of LoVo colon cancer cells through PRL-3-integrin beta1-ERK1/2 and-MMP2 signaling. Mol Cancer 8, 110

14. Radke, I., Gotte, M., Smollich, M., Scharle, N., Kiesel, L., and Wulfing, P. (2017) Expression of PRL-3 regulates proliferation and invasion of breast cancer cells in vitro. Arch Gynecol Obstet 296, 1153-1160

15. Zeng, Q., Hong, W., and Tan, Y. H. (1998) Mouse PRL-2 and PRL-3, two potentially prenylated protein tyrosine phosphatases homologous to PRL-1. Biochem Biophys Res Commun 244, 421-427

16. Zimmerman, M. W., Homanics, G. E., and Lazo, J. S. (2013) Targeted deletion of the metastasis-associated phosphatase Ptp4a3 (PRL-3) suppresses murine colon cancer. PLoS One 8, e58300

17. Wei, M., Korotkov, K. V., and Blackburn, J. S. (2018) Targeting phosphatases of regenerating liver (PRLs) in cancer. Pharmacol Ther 190, 128-138

18. Hoeger, B., Diether, M., Ballester, P. J., and Kohn, M. (2014) Biochemical evaluation of virtual screening methods reveals a cell-active inhibitor of the cancer-promoting phosphatases of regenerating liver. Eur J Med Chem 88, 89-100

19. Daouti, S., Li, W. H., Qian, H., Huang, K. S., Holmgren, J., Levin, W., Reik, L., McGady, D. L., Gillespie, P., Perrotta, A., Bian, H., Reidhaar-Olson, J. F., Bliss, S. A., Olivier, A. R., Sergi, J. A., Fry, D., Danho, W., Ritland, S., Fotouhi, N., Heimbrook, D., and Niu, H. (2008) A selective phosphatase of regenerating liver phosphatase inhibitor suppresses tumor cell anchorage-independent growth by a novel mechanism involving p130Cas cleavage. Cancer Res 68, 1162-1169

20. McQueeney, K. E., Salamoun, J. M., Burnett, J. C., Barabutis, N., Pekic, P., Lewandowski, S. L., Llaneza, D. C., Cornelison, R., Bai, Y., Zhang, Z. Y., Catravas, J. D., Landen, C. N., Wipf, P., Lazo, J. S., and Sharlow, E. R. (2018) Targeting ovarian cancer and endothelium with an allosteric PTP4A3 phosphatase inhibitor. Oncotarget 9, 8223-8240

21. Ooki, A., Yamashita, K., Kikuchi, S., Sakuramoto, S., Katada, N., and Watanabe, M. (2010) Phosphatase of regenerating liver-3 as a convergent therapeutic target for lymph node metastasis in esophageal squamous cell carcinoma. Int J Cancer 127, 543-554

22. Thura, M., Al-Aidaroos, A. Q., Gupta, A., Chee, C. E., Lee, S. C., Hui, K. M., Li, J., Guan, Y. K., Yong, W. P., So, J., Chng, W. J., Ng, C. H., Zhou, J., Wang, L. Z., Yuen, J. S. P., Ho, H. S. S., Yi, S. M., Chiong, E., Choo, S. P., Ngeow, J., Ng, M. C. H., Chua, C., Yeo, E. S. A., Tan, I. B. H., Sng, J. X. E., Tan, N. Y. Z., Thiery, J. P., Goh, B. C., and Zeng, Q. (2019) PRL3-zumab as an immunotherapy to inhibit tumors expressing PRL3 oncoprotein. Nat Commun 10, 2484

23. Allegra, A., Innao, V., Gerace, D., Vaddinelli, D., Allegra, A. G., and Musolino, C. (2018) Nanobodies and Cancer: Current Status and New Perspectives. Cancer Invest 36, 221 237

24. Bannas, P., Hambach, J., and Koch-Nolte, F. (2017) Nanobodies and Nanobody-Based Human Heavy Chain Antibodies As Antitumor Therapeutics. Front Immunol 8, 1603

25. Muyldermans, S. (2001) Single domain camel antibodies: current status. J Biotechnol 74, 277-302 
26. Krah, S., Schroter, C., Zielonka, S., Empting, M., Valldorf, B., and Kolmar, H. (2016) Single-domain antibodies for biomedical applications. Immunopharmacol Immunotoxicol 38, 21-28

27. Schmitz, K. R., Bagchi, A., Roovers, R. C., van Bergen en Henegouwen, P. M., and Ferguson, K. M. (2013) Structural evaluation of EGFR inhibition mechanisms for nanobodies/VHH domains. Structure 21, 1214-1224

28. Yang, E. Y., and Shah, K. (2020) Nanobodies: Next Generation of Cancer Diagnostics and Therapeutics. Front Oncol 10, 1182

29. Muruganandam, A., Tanha, J., Narang, S., and Stanimirovic, D. (2002) Selection of phagedisplayed llama single-domain antibodies that transmigrate across human blood-brain barrier endothelium. FASEB J 16, 240-242

30. Muyldermans, S., Atarhouch, T., Saldanha, J., Barbosa, J. A., and Hamers, R. (1994) Sequence and structure of VH domain from naturally occurring camel heavy chain immunoglobulins lacking light chains. Protein Eng 7, 1129-1135

31. Chow, K. M., Whiteheart, S. W., Smiley, J. R., Sharma, S., Boaz, K., Coleman, M. J., Maynard, A., Hersh, L. B., and Vander Kooi, C. W. (2019) Immunization of Alpacas (Lama pacos) with Protein Antigens and Production of Antigen-specific Single Domain Antibodies. J Vis Exp

32. Lian, S., Meng, L., Yang, Y., Ma, T., Xing, X., Feng, Q., Song, Q., Liu, C., Tian, Z., Qu, L., and Shou, C. (2017) PRL-3 promotes telomere deprotection and chromosomal instability. Nucleic Acids Res 45, 6546-6571

33. Zeng, Q., Si, X., Horstmann, H., Xu, Y., Hong, W., and Pallen, C. J. (2000) Prenylationdependent association of protein-tyrosine phosphatases PRL-1, -2 , and -3 with the plasma membrane and the early endosome. J Biol Chem 275, 21444-21452

34. Thura, M., Al-Aidaroos, A. Q. O., Yong, W. P., Kono, K., Gupta, A., Lin, Y. B., Mimura, K., Thiery, J. P., Goh, B. C., Tan, P., Soo, R., Hong, C. W., Wang, L., Lin, S. J., Chen, E., Rha, S. Y., Chung, H. C., Li, J., Nandi, S., Yuen, H. F., Zhang, S. D., Guan, Y. K., So, J., and Zeng, Q. (2016) PRL3-zumab, a first-in-class humanized antibody for cancer therapy. JCI Insight 1, e87607

35. Herce, H. D., Schumacher, D., Schneider, A. F. L., Ludwig, A. K., Mann, F. A., Fillies, M., Kasper, M. A., Reinke, S., Krause, E., Leonhardt, H., Cardoso, M. C., and Hackenberger, C. P. R. (2017) Cell-permeable nanobodies for targeted immunolabelling and antigen manipulation in living cells. Nat Chem 9, 762-771

36. Nischan, N., Herce, H. D., Natale, F., Bohlke, N., Budisa, N., Cardoso, M. C., and Hackenberger, C. P. (2015) Covalent attachment of cyclic TAT peptides to GFP results in protein delivery into live cells with immediate bioavailability. Angew Chem Int Ed Engl 54, 1950-1953

37. Huo, J., Le Bas, A., Ruza, R. R., Duyvesteyn, H. M. E., Mikolajek, H., Malinauskas, T., Tan, T. K., Rijal, P., Dumoux, M., Ward, P. N., Ren, J., Zhou, D., Harrison, P. J., Weckener, M., Clare, D. K., Vogirala, V. K., Radecke, J., Moynie, L., Zhao, Y., Gilbert-Jaramillo, J., Knight, M. L., Tree, J. A., Buttigieg, K. R., Coombes, N., Elmore, M. J., Carroll, M. W., Carrique, L., Shah, P. N. M., James, W., Townsend, A. R., Stuart, D. I., Owens, R. J., and Naismith, J. H. (2020) Neutralizing nanobodies bind SARS-CoV-2 spike RBD and block interaction with ACE2. Nat Struct Mol Biol 27, 846-854 
38. Tabtimmai, L., Suphakun, P., Srisook, P., Kiriwan, D., Phanthong, S., Kiatwuthinon, P., Chaicumpa, W., and Choowongkomon, K. (2019) Cell-penetrable nanobodies (transbodies) that inhibit the tyrosine kinase activity of EGFR leading to the impediment of human lung adenocarcinoma cell motility and survival. J Cell Biochem 120, 1807718087

39. Dekempeneer, Y., Back, T., Aneheim, E., Jensen, H., Puttemans, J., Xavier, C., Keyaerts, M., Palm, S., Albertsson, P., Lahoutte, T., Caveliers, V., Lindegren, S., and D'Huyvetter, M. (2019) Labeling of Anti-HER2 Nanobodies with Astatine-211: Optimization and the Effect of Different Coupling Reagents on Their in Vivo Behavior. Mol Pharm 16, 35243533

40. Schriewer, L., Schutze, K., Petry, K., Hambach, J., Fumey, W., Koenigsdorf, J., Baum, N., Menzel, S., Rissiek, B., Riecken, K., Fehse, B., Rockendorf, J. L., Schmid, J., Albrecht, B., Pinnschmidt, H., Ayuk, F., Kroger, N., Binder, M., Schuch, G., Hansen, T., Haag, F., Adam, G., Koch-Nolte, F., and Bannas, P. (2020) Nanobody-based CD38-specific heavy chain antibodies induce killing of multiple myeloma and other hematological malignancies. Theranostics 10, 2645-2658 